\title{
Staphylococcus aureus drives expansion of low-density neutrophils in diabetic mice
}

\author{
Taylor S. Cohen, ${ }^{1}$ Virginia Takahashi, ${ }^{1}$ Jessica Bonnell, ${ }^{1}$ Andrey Tovchigrechko, ${ }^{2}$ Raghothama Chaerkady, ${ }^{3}$ Wen Yu, ${ }^{4}$ \\ Omari Jones-Nelson, ${ }^{1}$ Young Lee, ${ }^{2}$ Rajiv Raja, ${ }^{2}$ Sonja Hess, ${ }^{3}$ C. Kendall Stover, ${ }^{1}$ John J. Worthington, ${ }^{5}$ Mark A. Travis, ${ }^{6,7,8}$ \\ and Bret R. Sellman' \\ 1Department of Microbial Sciences, ${ }^{2}$ Department of Translational Medicine and Pharmacogenetics, ${ }^{3}$ Department of Antibody Discovery and Protein Engineering, and ${ }^{4}$ Bioinformatics, AstraZeneca, \\ Gaithersburg, Maryland, USA. ${ }^{5}$ Biomedical and Life Sciences, Faculty of Health and Medicine, University of Lancaster, Lancaster, United Kingdom. ${ }^{6}$ Lydia Becker Institute of Immunology and Inflammation, \\ ${ }^{7}$ Manchester Collaborative Centre for Inflammation Research, Faculty of Biology, Medicine and Health, Manchester Academic Health Science Centre, and ${ }^{8}$ Wellcome Trust Centre for Cell-Matrix Research, \\ University of Manchester, Manchester, United Kingdom.
}

\begin{abstract}
Diabetic individuals are at considerable risk for invasive infection by Staphylococcus aureus, however, the mechanisms underlying this enhanced susceptibility to infection are unclear. We observed increased mortality following i.v. S. aureus infection in diabetic mice compared with nondiabetic controls, correlating with increased numbers of low-density neutrophils (LDNs) and neutrophil extracellular traps (NETs). LDNs have been implicated in the inflammatory pathology of diseases such as lupus, given their release of large amounts of NETs. Our goal was to describe what drives LDN increases during $S$. aureus infection in the diabetic host and mechanisms that promote increased NET production by LDNs. LDN development is dependent on TCF- $\beta$, which we found to be more activated in the diabetic host. Neutralization of TCF- $\beta$, or the TCF- $\beta$ activating integrin $\alpha_{v} \beta_{8}$, reduced LDN numbers and improved survival during $S$. aureus infection. Targeting $S$. aureus directly with MEDI4893*, an $\alpha$ toxin-neutralizing monoclonal antibody, blocked TCF- $\beta$ activation, reduced LDNs and NETs, and significantly improved survival. A comparison of gene and protein expression in high-density neutrophils and LDNs identified increased CPCRs and elevated phosphatase and tensin homolog (PTEN) in the LDN subset. Inhibition of PTEN improved the survival of infected diabetic mice. Our data identify a population of neutrophils in infected diabetic mice that correlated with decreased survival and increased NET production and describe 3 therapeutic targets, a bacterial target and 2 host proteins, that prevented NET production and improved survival.
\end{abstract}

\section{Introduction}

Staphylococcus aureus is a major human pathogen responsible for causing numerous types of human disease. Defense against $S$. aureus infection requires a highly coordinated innate immune response, including cytokine production by resident cells, production and release of antimicrobial peptides, recruitment of phagocytes, and phagocytic killing of the bacteria. Proper coordination requires a balance of inflammatory and antiinflammatory signals, not only to recruit the appropriate cells but also to prevent inflammatory tissue damage. Alterations in this balance can render the host incredibly susceptible to infection with opportunistic pathogens such as $S$. aureus.

Diseases associated with chronic inflammation, such as diabetes, are linked to an increased risk of opportunistic infections (1-3). Although the nature of the diabetic immune defect has yet to be fully elucidated, murine models of diabetic infection pointed

Conflict of interest: TSC, VT, JB, AT, RC, WY, OJN, YL, RR, SH, CKS, and BRS are employees of AstraZeneca. JJW and MAT received antibodies from AstraZeneca to perform studies.

Copyright: () 2019, American Society for Clinical Investigation.

Submitted: December 18, 2018; Accepted: March 5, 2019; Published: April 15, 2019

Reference information: J Clin Invest. 2019;129(5):2133-2144.

https://doi.org/10.1172/JCl126938. to defective respiratory burst in neutrophils isolated from diabetic mice as a potential mechanism through which elevated glucose impairs innate immune function (4). Similar defects were reported in a study of neutrophils from patients with type 1 diabetes, whereas phagocytic function remained intact $(5,6)$.

Neutrophils have been implicated in the heightened inflammatory state in the diabetic host. In mice, a reduction in neutrophil clearance by macrophages and slower rates of neutrophil apoptosis led to prolonged release of inflammatory cytokines such as TNF- $\alpha$ (7). In addition to cytokines, neutrophils release DNA strands coated with antimicrobial peptides, which are structures known as neutrophil extracellular traps (NETs) $(8,9)$. Originally thought to have antimicrobial function, more recent data showing recovery of viable bacteria from DNAse-disrupted NETs and damage to endothelial cells suggest a more pathogenic and less bactericidal nature of NET release (10-12). In the context of diabetes, analysis of patients' serum has shown increased NET levels in patients with early-onset type 1 diabetes, as well as increased NETs visualized in the wounds of diabetic mice (13-16).

In this study, we identify a subset of neutrophils, low-density neutrophils (LDNs), that increase in diabetic mice during infection. Increases in this cell population were not observed in nondiabetic control mice when infected with $S$. aureus. We found that TGF- $\beta$, activated in response to $S$. aureus $\alpha$ toxin (AT) and integrin 
A

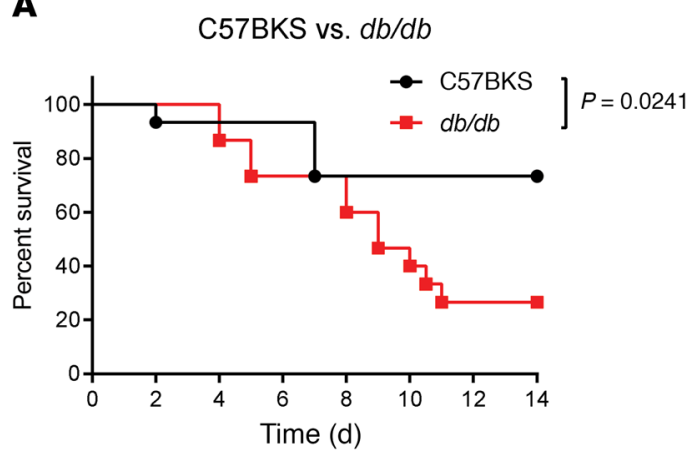

C

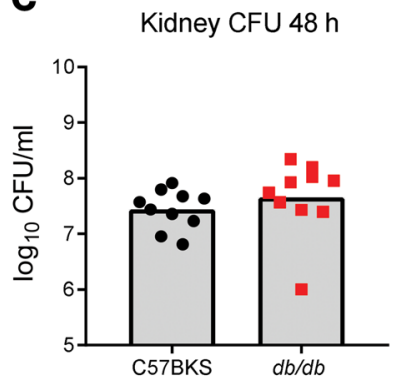

$\mathbf{F}$

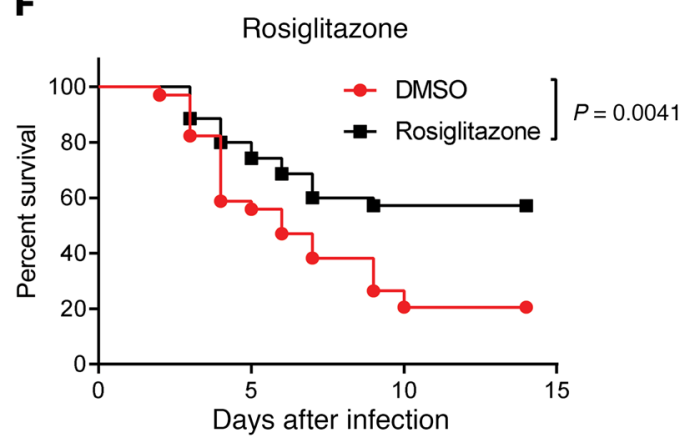

B

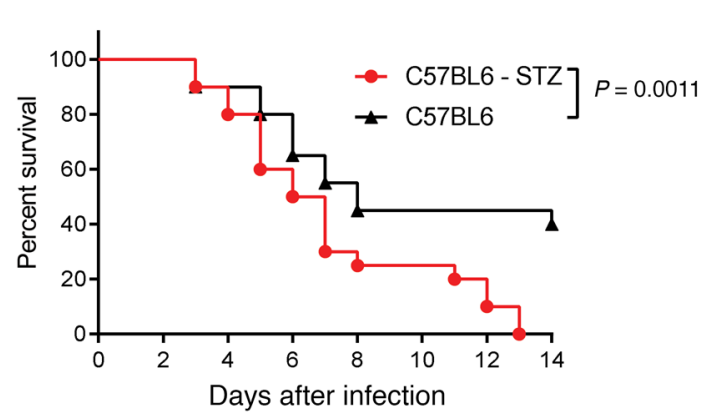

E
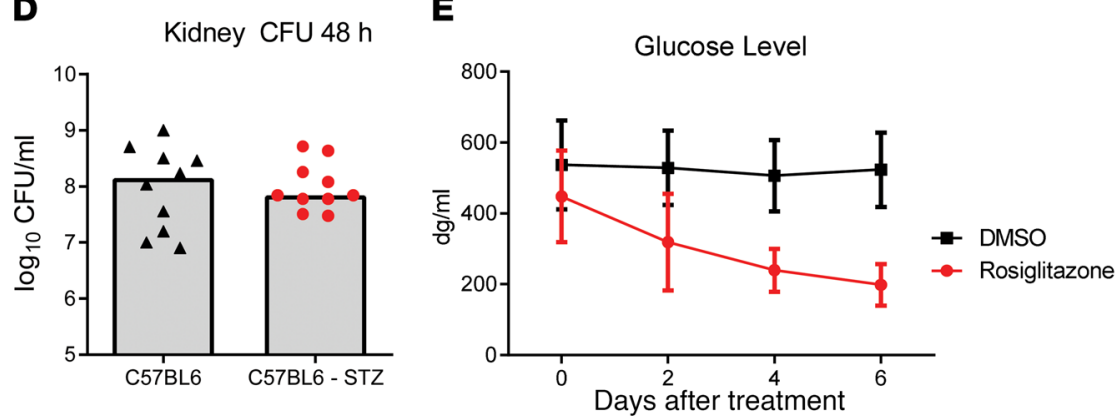

G

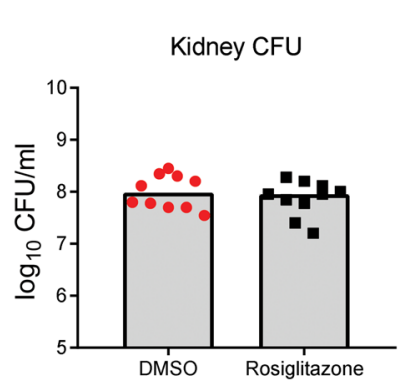

Figure 1. Diabetic mice are more susceptible to infection with S. aureus. (A) Survival of C57BKS and $d b / d b$ mice infected i.v. with $5 \times 10^{7}$ CFU S. aureus ( $n=15$ mice per group). (B) Survival of C57BL/6 control or STZ-treated mice infected i.v. with $5 \times 10^{7}$ CFU S. aureus ( $n=20$ mice per group). (C and $\left.\mathbf{D}\right)$ Numbers of $S$. aureus CFU recovered from the kidneys 48 hours after infection. (E) Glucose levels in the blood of mice treated with rosiglitazone or DMSO ( $n=10$ mice per group). (F) Survival of $d b / d b$ mice treated with rosiglitazone or DMSO for 7 days prior to infection with $5 \times 10^{7}$ CFU S. aureus ( $n=20$ mice per group). (C) Numbers of S. aureus CFU recovered from the kidneys of $d b / d b$ mice 48 hours after infection. All data are representative of at least 3 independent experiments. Statistical significance was determined by log-rank test (A, B, and $\mathbf{F})$ and Mann-Whitney $U$ test (C, D, and $\mathbf{C})$. Bars indicate the median.

$\alpha_{\mathrm{v}} \beta_{8}$, was responsible for increased LDNs during infection. Additionally, we identified a key signaling cascade involving phosphatase and tensin homolog (PTEN) that is altered in the LDN population. Inhibition of PTEN resulted in improved survival of diabetic mice during infection with $S$. aureus. These data highlight potential therapeutic targets and identify mechanisms that promote opportunistic infections in individuals with diabetes.

\section{Results}

Elevated glucose levels correlate with more severe S. aureus infection. We used 2 murine diabetes models, streptozotocin-induced (STZinduced) (type 1 diabetes) and obese $d b / d b$ (type 2 diabetes), to study the effect of diabetes on the systemic response to a $S$. aureus bloodstream infection. In each model, diabetic mice had a nonfasting glucose level greater than $450 \mathrm{dg} / \mathrm{ml}$, whereas levels in the nondiabetic control mice were less than $200 \mathrm{dg} / \mathrm{ml}$. Mice were infected i.v. with $5 \times 10^{7}$ CFU S. aureus (USA300, SF8300). CFU were enumerated in the kidneys 48 hours after infection, and mortality was monitored for 14 days. We observed increased mortality in both the STZ $(P=0.0011)$ and $d b / d b(P=0.0241)$ models as compared with mortality rates for the nondiabetic controls (Figure 1, A and B). Of note, this enhanced mortality did not correlate with a difference in bacterial CFU recovered from the kidneys 48 hours after infection (Figure 1, C and D). To confirm that increased mortality was a consequence of elevated glucose in the diabetic host, we treated $d b / d b$ mice with the insulin-sensitizing PPAR $\gamma$ agonist rosiglitazone for 1 week prior to infection to reduce circulating glucose levels (Figure 1E). We found that rosiglitazone significantly reduced mortality $(P=0.0041)$ following infection with $S$. aureus, without affecting bacterial burden in the kidney (Figure 1, F and G).

Enhanced NET release in diabetic mice. Neutrophils in a diabetic host, or in the presence of elevated glucose levels, are increasingly 

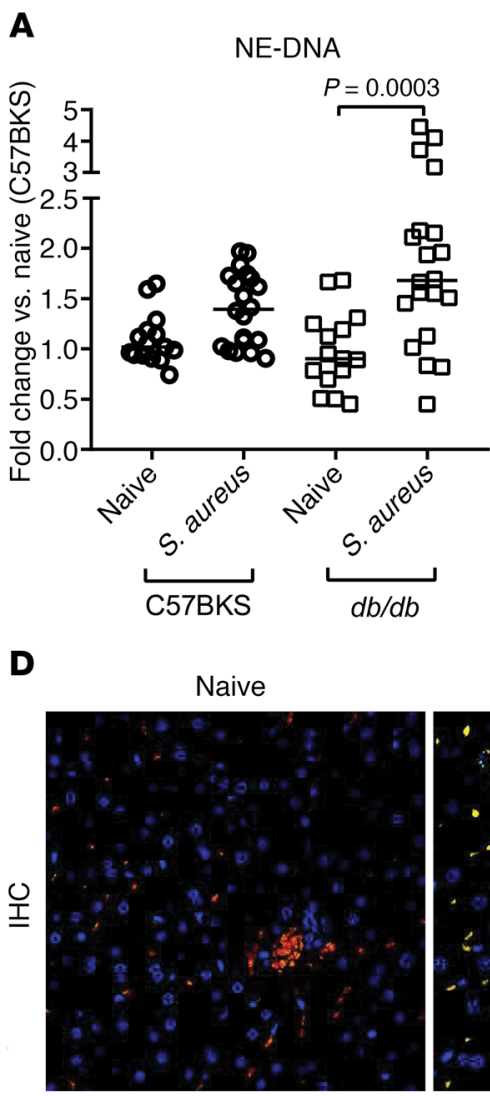

B

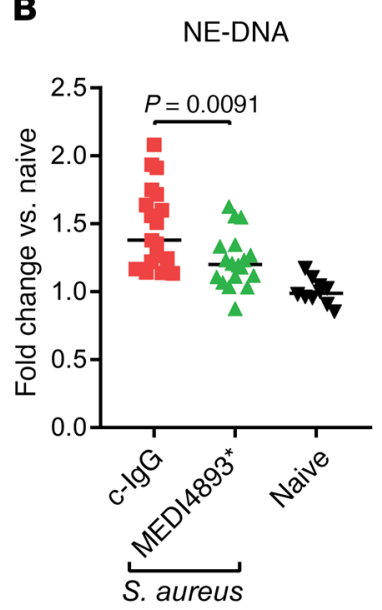

C Liver $\mathrm{H}_{3} \mathrm{Cit}$

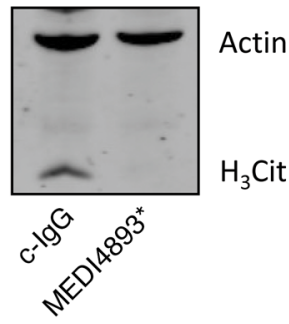

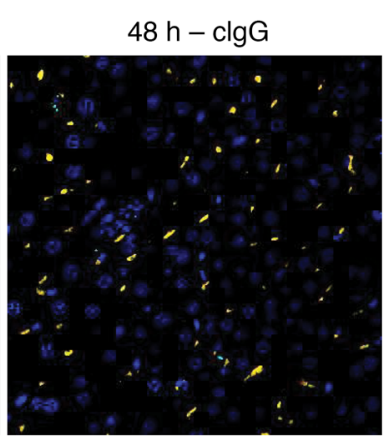

Ly6-G

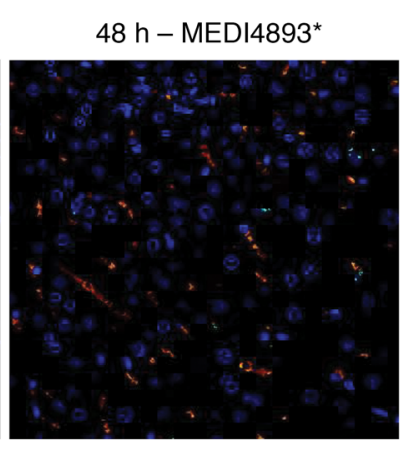

$\mathbf{E}$

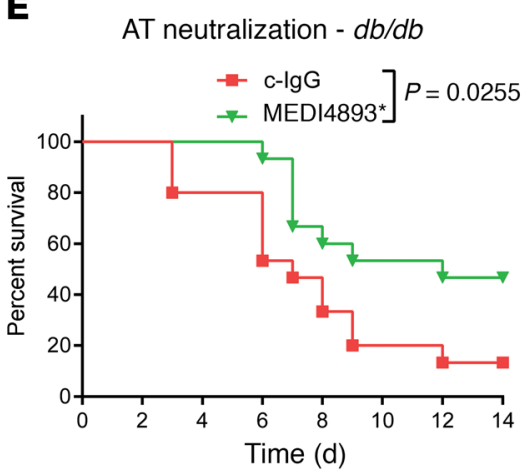

Colocalized (yellow)

Figure 2. NET induction in response to S. aureus. (A) ELISA measurement of NETs in the serum of C57BKS and $d b / d b$ mice with or without infection $(24 \mathrm{~h})$ with S. aureus. (B) ELISA measurement of NETs in the serum of $d b / d b$ mice treated with MEDI4893* or c-lgG and infected with S. aureus (24 h). (C) Western blot of liver tissue taken from mice treated with MEDI4893* or c-lgG 24 hours prior to infection (48 h) with S. aureus. (D) Confocal micrographs of livers isolated from naive or infected $d b / d b$ mice 48 hours after infection with S. aureus. Original magnification, $\times 40$. (E) Survival of $d b / d b$ mice treated with MEDI4893* or c-IgC 24 hours prior to infection with $5 \times 10^{7}$ CFU S. aureus ( $n=15$ mice per group). All data are representative of at least 3 independent experiments. $n \geq 10$ per group (A and B); $n \geq 5$ per group (C and $\mathbf{D}$ ). Statistical significance was determined by Mann-Whitney $U$ test (A and B) (bars represent the median) and log-rank test $(\mathbf{E})$.

prone to forming NETs, which are composed of DNA, citrullinated histones, enzymes, and antimicrobial peptides. In the diabetic population, NET release has been shown to impair wound healing in mice, and the presence of NETs in the serum correlates with nonhealing wounds in patients $(15,16)$. As neutrophils also release NETs in response to bacterial infection, we hypothesized that $S$. aureus infection would result in increased systemic NET release in diabetic mice. Complexes of neutrophil elastase (NE) and double-stranded DNA have been used as a measurement of NET formation and quantified by ELISA (15). We observed significant increases $(P=0.0003)$ in serum NE-DNA complexes in diabetic mice infected i.v. with $S$. aureus for 24 hours, whereas significant increases were not observed in the nondiabetic control mice (Figure 2A). AT, once released by $S$. aureus, binds to ADAM10 on the surface of platelets. AT-bound platelets induce NET production when bound to circulating neutrophils (17). Consistent with these findings, neutralization of AT with the monoclonal antibody MEDI4893* significantly reduced the number of NE-DNA complexes in the serum 48 hours after infection in diabetic animals
(Figure 2B). Increased AT-dependent NET production was confirmed 48 hours after infection by increased citrullinated histone $\mathrm{H} 3\left(\mathrm{H}_{3} \mathrm{Cit}\right)$ in the liver as detected by Western blotting and visualization of liver sections stained with anti-Ly6 G to mark neutrophils and anti- $\mathrm{H}_{3}$ Cit (Figure 2, C and D). Importantly, neutralization of AT significantly increased the survival $(P=0.0255)$ of diabetic mice, but not nondiabetic control mice, infected with $S$. aureus (Figure 2E and Supplemental Figure 1; supplemental material available online with this article; https://doi.org/10.1172/JCI126938DS1). We concluded that systemic infection of the diabetic host led to an AT-dependent increase in circulating NETs, with therapeutic blockade of AT significantly improving survival after infection.

LDNs correlate with increased NET release. Like macrophages, neutrophils can be separated into different classes on the basis of functional characteristics. Tsuda et al. demonstrated that a severe burn altered the phenotype of circulating neutrophils, altering TLR expression, cytokine production, and their ability to drive macrophage polarization (18). An altered neutrophil phenotype correlated with an inability of the animals to survive $S$. aureus 
A
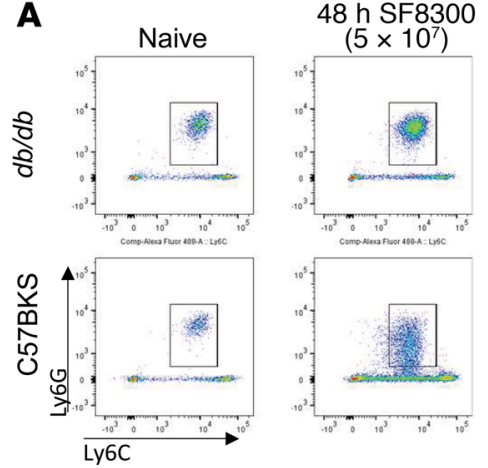

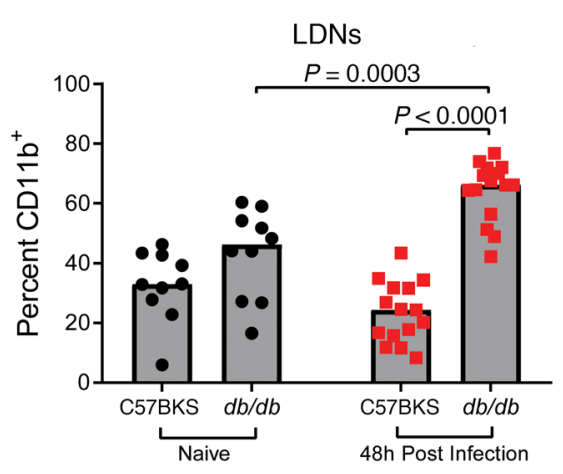

E

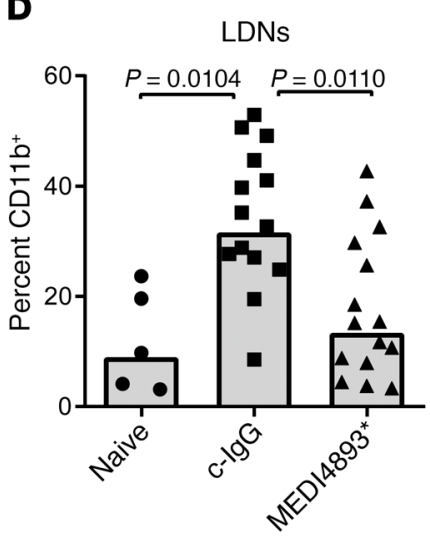

B

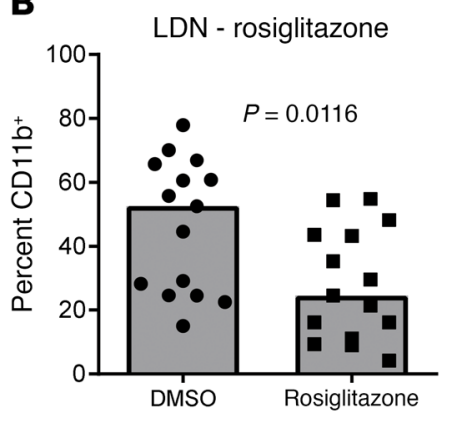

c

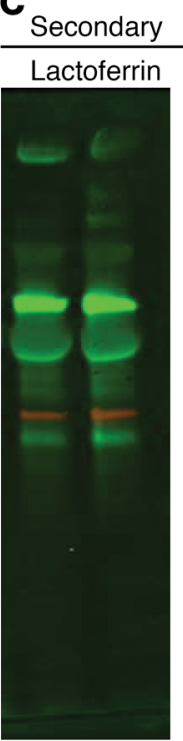

LD HD
Tertiary

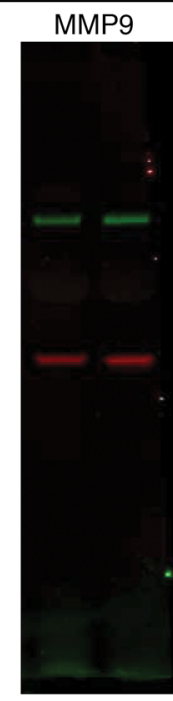

LD HD

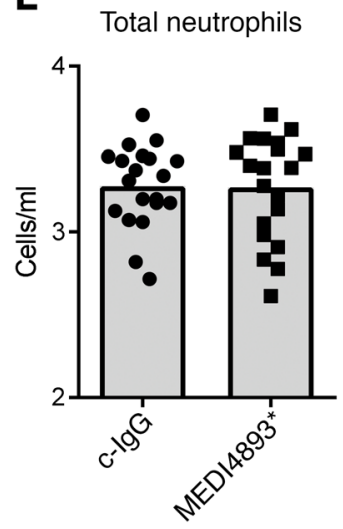

Figure 3. LDNs in diabetic mice. (A) FACS analysis of $C D 45^{+}{ }^{+} D 11 b^{+}$cells in the blood of C57BKS and $d b / d b$ mice showed increased LDNs in infected $d b / d b$ mice. (B) FACS analysis of LDNs in the blood of $d b / d b$ mice treated with DMSO or rosiglitazone and infected with S. aureus for 48 hours. (C) Western blot analysis of secondary and tertiary granule content in HDNs and LDNs isolated from $d b / d b$ mice 48 hours after infection. (D) FACS analysis of LDNs in the blood of naive $d b / d b$ mice or $d b / d b$ mice treated with MEDI4893* or C-IgC 24 hours prior to infection (48 h) with S. aureus. (E) FACS analysis of the neutrophil numbers in the blood of mice treated with MEDI4893* or c-IgG 24 hours prior to infection ( $48 \mathrm{~h}$ ) with S. aureus. All data representative of at least 3 independent experiments. $n \geq 10$ per group (A, B, D, and E); $n \geq 5$ per group (C). Statistical significance was determined by Kruskal-Wallis followed by Dunn's test (A and $\mathbf{D}$ ) and Mann-Whitney $U$ test (B and $\mathbf{E}$ ). All data are represented as individual points, with the bar representing the median. infection. Neutrophils can also be separated by density. Highdensity neutrophils (HDNs) are antitumor, phagocytic cells, whereas LDNs are considered protumor, phagocytosis-defective cells (19). Although Tsuda et al. did not measure the density of neutrophils isolated from $S$. aureus-susceptible mice, the shape of the nuclei in these neutrophils was similar to that of nuclei in low-density cells $(19,20)$. We found striking differences in the shapes of the nuclei in neutrophils taken from nondiabetic mice and diabetic mice, with the nuclei in cells isolated from diabetic mice more closely resembling the ringed nuclei reported in LDNs (Supplemental Figure 2).

Hyperproduction of NETs is a characteristic of LDNs, and we hypothesized that higher numbers of LDNs in infected diabetic mice were responsible for the increases in NETs (21). To test this hypothesis, we collected blood from C57BKS and $d b / d b$ mice 48 hours after i.v. infection and analyzed it for the presence of LDNs. The amount of LDNs in the blood of infected $d b / d b$ mice was significantly increased compared with numbers in the blood of uninfected $d b / d b$ mice $(P<0.0001)$ as well as infected C57BKS control mice $(P=0.0003)$ (Figure $3 \mathrm{~A})$. Increases in LDNs were not observed in C57BKS mice following infection (Figure 3A). We observed similar increases in STZ-induced diabetic mice but not in C57BL/6 control mice (Supplemental Figure 3). Enhancement of LDNs after infection was dependent on glucose levels, as lowering glucose levels with rosiglitazone prior to infection significantly $(P=0.0116)$ reduced LDN numbers 48 hours after infection (Figure 3B).

To ensure that we were not simply observing degranulated neutrophils, we isolated LDNs and HDNs from the blood of infected $d b / d b$ mice and measured the amount of lactoferrin (secondary granules) and MMP9 (tertiary granules) by Western blotting. Consistent with previous work (19), we detected equivalent amounts of both lactoferrin and MMP9 in each cell type, indicating that LDNs have a granular content similar to that of HDNs (Figure 3C). Since neutralizing AT prevented systemic NET release (Figure 2B), we questioned whether AT influenced the number of LDNs. We measured LDNs in the blood of $d b / d b$ mice passively immunized with c-IgG or MEDI4893* twenty-four hours before infection with S. aureus. We observed a significant reduction in LDNs in mice prophylactically treated with MEDI4893* (Figure 3D), whereas the overall numbers of neutrophils were not affected (Figure 3E) 48 hours after infection, indicating that AT contributes to the increase in LDNs.

TGF- $\beta$ drives expansion of the LDN population. Next, we attempted to determine additional important mechanisms driving 
$\mathbf{A}$
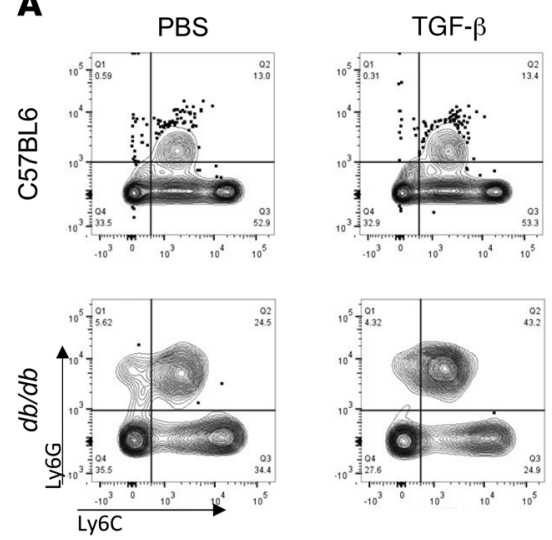

D

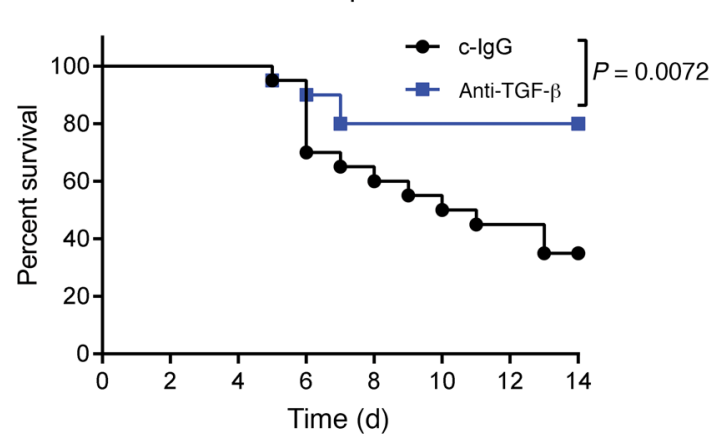

LDNs - C57BL/6

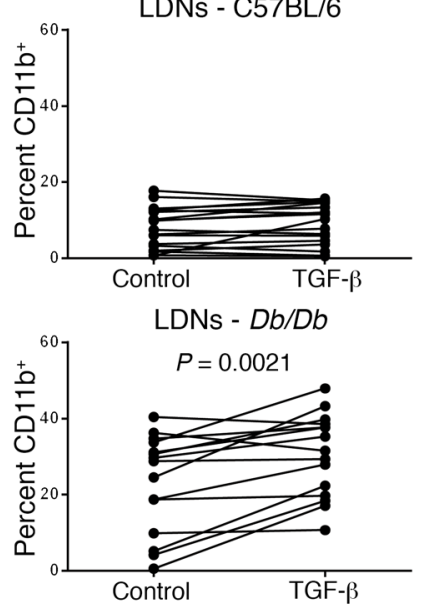

B

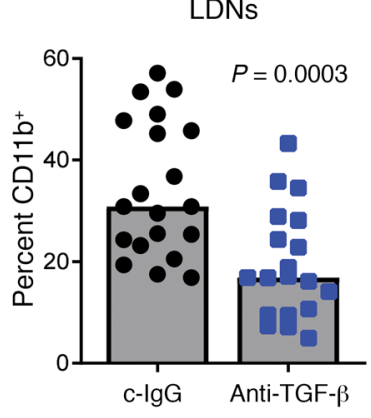

C

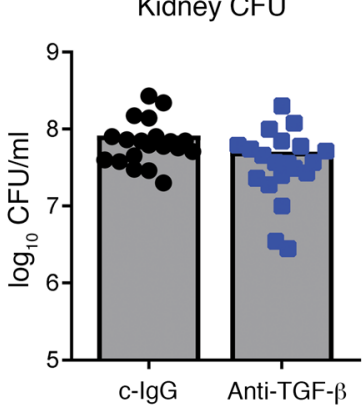

$\mathbf{E}$

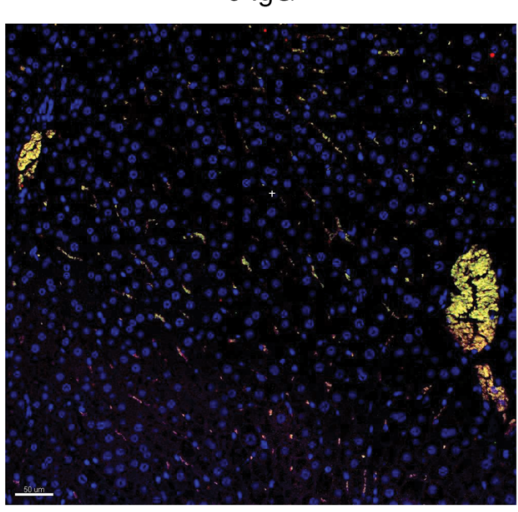

Ly6G

NET

Colocalized (yellow)

Figure 4. LDNs are driven by TGF- $\beta$ signaling. (A) FACS analysis of CD45+CD11 b+ cells in the low-density fraction of whole blood treated ex vivo with albumin or TCF- $\beta$. (B) FACS analysis of LDNs in the blood of $d b / d b$ mice treated with anti-TCF- $\beta$ or c-lgG and infected for 48 hours with S. aureus. Bars represent the median. (C) Numbers of S. aureus CFU recovered from the kidneys of $d b / d b$ mice treated with anti-TGF- $\beta$ or c-IgG and infected for 48 hours with S. aureus. Bars represent the median. (D) Survival of $d b / d b$ mice treated with anti-TGF- $\beta$ or c-lgG and infected for 14 days with S. aureus ( $n=20$ mice per group). (E) Confocal micrographs of liver tissue from mice 48 hours after infection with S. aureus. Original magnification, $\times 40$. All data are representative of at least 3 independent experiments. $n \geq 10$ per group (A-C); $n \geq 5$ per group (E). Statistical significance was determined by Mann-Whitney $U$ test (A-C) and log-rank test (D).

enhanced LDN and NET formation in diabetic mice. TGF- $\beta$ has been implicated as a central regulator of neutrophil phenotype and in tumor models can drive a phenotypic switch from HDNs to LDNs $(19,20)$. To test the importance of TGF- $\beta$ in the regulation of neutrophils in diabetic mice, we treated blood from nondiabetic and diabetic mice with TGF- $\beta$. We observed that addition of TGF- $\beta$ to diabetic blood significantly increased $(P=0.0021)$ the number of LDNs, whereas there was no difference in LDNs when TGF- $\beta$ was added to the blood of nondiabetic mice (Figure $4 \mathrm{~A}$ ). Additionally, prophylactic treatment of diabetic mice with a TGF- $\beta$ neutralizing antibody 24 hours prior to infection with $S$. aureus resulted in a significant reduction $(P=0.0003)$ in the number of LDNs in the bloodstream, whereas bacteria numbers in the kidneys were similar between the groups (Figure 4, B and C). Survival was also significantly improved $(P=0.0072)$ by neutralizing TGF- $\beta$
(Figure 4D). Visualization of $\mathrm{H}_{3}$ Cit in the liver revealed a loss of NETs when TGF- $\beta$ was neutralized (Figure $4 \mathrm{E}$ ). Thus, these data suggest that TGF- $\beta$ promotes LDN formation and that reducing LDNs by blocking TGF- $\beta$ could promote survival after infection.

Activation of TGF- $\beta$ by integrin $\alpha_{1} \beta_{8}$ drives LDN formation. TGF- $\beta$ is secreted as a pro-form protein that needs to be activated in order to function. Binding of pro-TGF- $\beta$ by $\alpha_{\mathrm{v}} \beta_{8}$ integrin on DCs has been linked to its activation (22). To determine whether $S$. aureus infection influences the expression of $\alpha_{v} \beta_{8}$ integrin, we isolated innate immune cells from the livers of C57BKS and $d b / d b$ mice 24 hours after infection and analyzed the expression of $\alpha_{v} \beta_{8}$ by flow cytometry (Supplemental Figure 4). The numbers of integrin $\beta_{8}{ }^{+}$inflammatory monocytes and DCs increased significantly $(P=$ 0.0015 and $P<0.0001$, respectively) in the livers of $d b / d b$ mice, but not C57BKS mice, following infection (Figure 5A). Interest- 
A
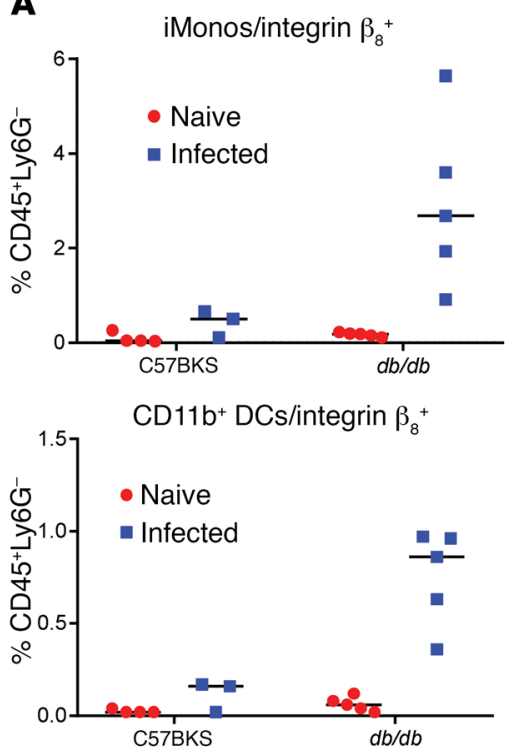

C

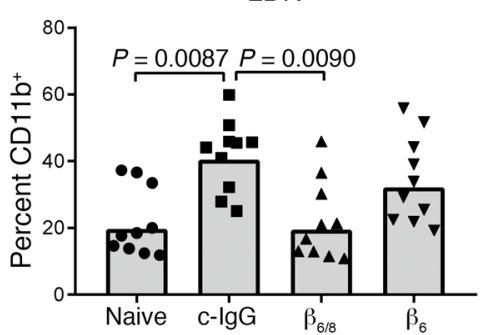

B
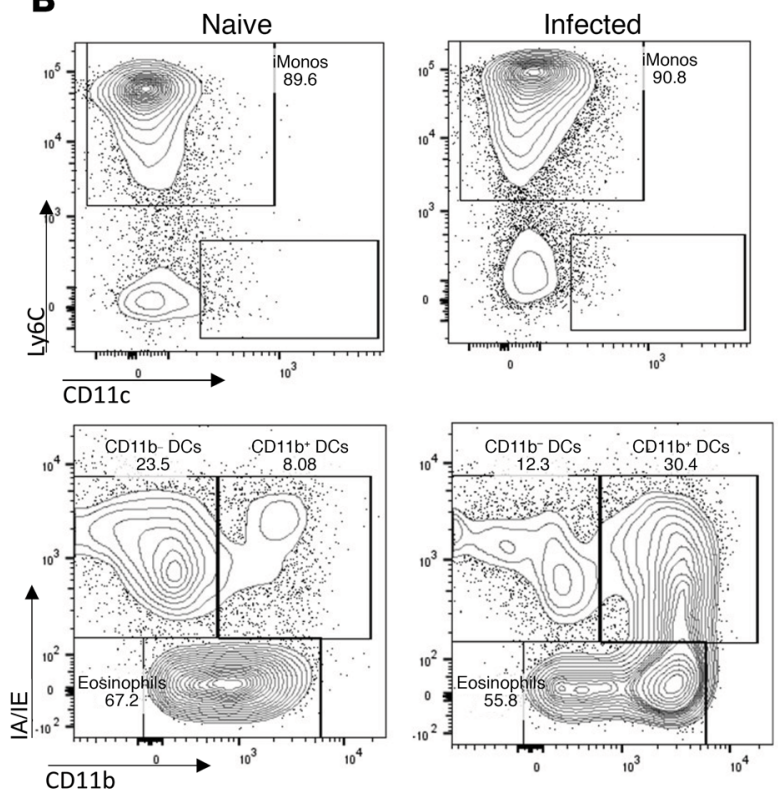

$\mathrm{B}_{8}$ expression - iMonocytes

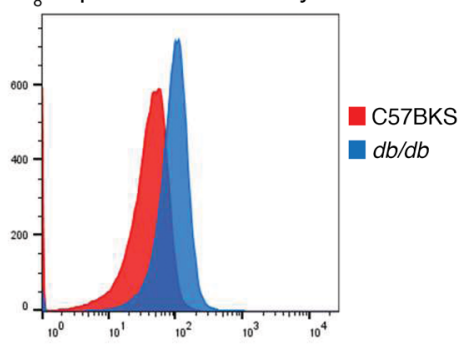

$\mathrm{B}_{8}$ expression - CD11b+ DCs

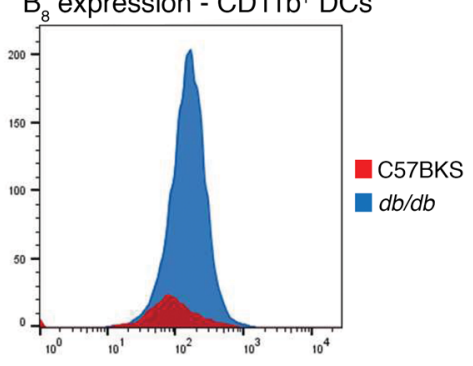

D

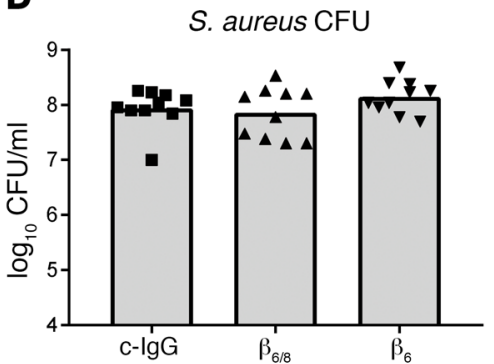

$\mathbf{E}$

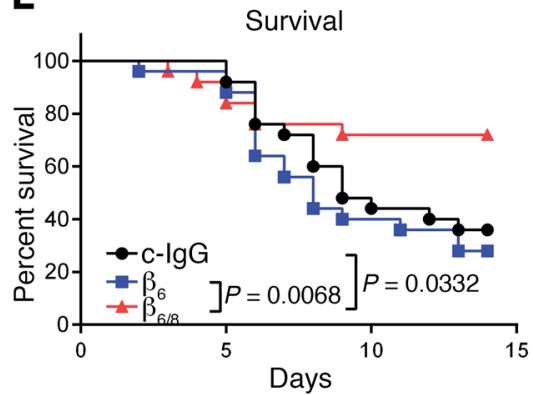

Figure 5. $\boldsymbol{\alpha}_{v} \beta_{8}$ Activity leads to expansion of the LDN population. (A) FACS analysis of $\beta_{8}$ expression on inflammatory monocytes (iMonos) and DCs in the liver. (B) FACS analysis of $\beta_{8}$ increased on cells from $d b / d b$ mice following 24 hours of infection with $S$. aureus. (C) FACS analysis of LDNs in the blood of $d b / d b$ mice 48 hours after infection with S. aureus. Bars represent the median. (D) Numbers of S. aureus CFU recovered from the kidneys of $d b / d b$ mice 48 hours after infection. Bars represent the median. (E) Survival of $d b / d b$ mice following infection with S. aureus ( $n=20$ per group). All data are representative of at least 3 independent experiments. $n=5$ per group (A); $n \geq 10$ per group (C-E). Statistical significance was determined by Mann-Whitney $U$ test (A), Kruskal-Wallis followed by Dunn's test (C and $\mathbf{D})$, and log-rank test (E).

ingly, while integrin expression increased on the surface of monocytes, it was the overall number of DCs that increased, not the density of $\beta_{8}$ (Figure $5 \mathrm{~B}$ ).

To determine the functional relevance of integrin $\alpha_{\mathrm{v}} \beta_{8}$ in this model, mice were prophylactically treated with antibodies neutralizing $\alpha_{\mathrm{v}} \beta_{6 / 8}, \alpha_{\mathrm{v}} \beta_{6}$, or c-IgG and then infected with $S$. aureus. Forty-eight hours after infection, we found that LDN numbers were significantly decreased $(P=0.0090)$ in the bloodstream of mice treated with $\alpha_{\mathrm{v}} \beta_{6 / 8}$-neutralizing antibody compared with those treated with c-IgG (Figure 5C). Neutralization of $\alpha_{v} \beta_{6}$ alone did not reduce the numbers of these cells. We also noted that integrin inhibition did not affect the numbers of bacteria in the kidneys 48 hours after infection (Figure 5D). Survival was significantly improved in the mice treated with anti- $\alpha_{v} \beta_{6 / 8}$ antibody as compared with the c-IgG- or anti- $\alpha_{v} \beta_{6}-$ treated mice (Figure 5E). Therefore, consistent with direct neutralization of TGF- $\beta$, inhibition of the integrin responsible for activating this pathway improved survival in diabetic mice.

AT drives TGF- $\beta$ activation. As neutralization of either AT or TGF- $\beta$ blocked increases in LDNs, we hypothesized that AT influenced LDN numbers by affecting the TGF- $\beta$ signaling pathway. Activation of SMAD signaling is commonly used as a surrogate measurement of active TGF- $\beta$ signaling (23). We therefore analyzed phosphorylated SMAD (p-SMAD) levels in the lives of diabetic and nondiabetic mice infected $(24 \mathrm{~h})$ with $S$. aureus. We observed significantly increased levels of p-SMAD in the livers of infected diabetic mice compared with levels in naive diabetic $(P<0.0001)$ and infected nondiabetic $(P=0.0338)$ mice (Figure 6A). In diabetic mice, MEDI4893* significantly reduced $(P<$ $0.0001) \mathrm{p}$-SMAD levels in the liver, indicating that AT was contributing to the activation of TGF- $\beta$ signaling (Figure 6B). We found that neutralization of AT did not alter the numbers of $\alpha_{\mathrm{v}} \beta_{8}$ expressing innate immune cells (Figure 6C). Therefore, we conclude that AT influences the activation of TGF- $\beta$, although the mechanism is independent of $\alpha_{v} \beta_{8}$ expression on the surface of innate immune cells.

Altered gene and protein expression in LDNs. While our data indicate that AT activation of TGF- $\beta$ signaling is important for the increases in LDNs, we sought to better understand what leads to 
A

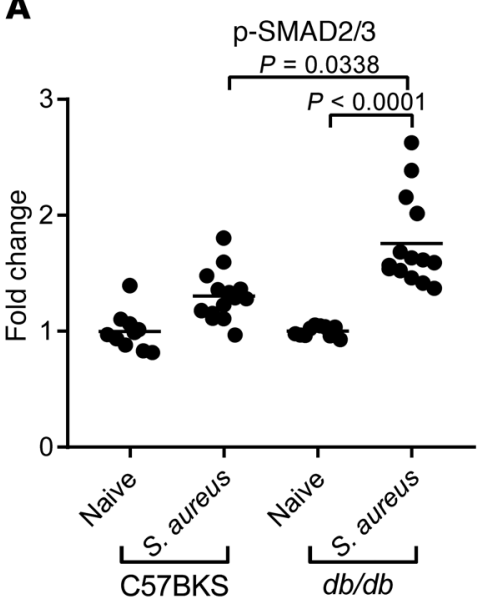

c

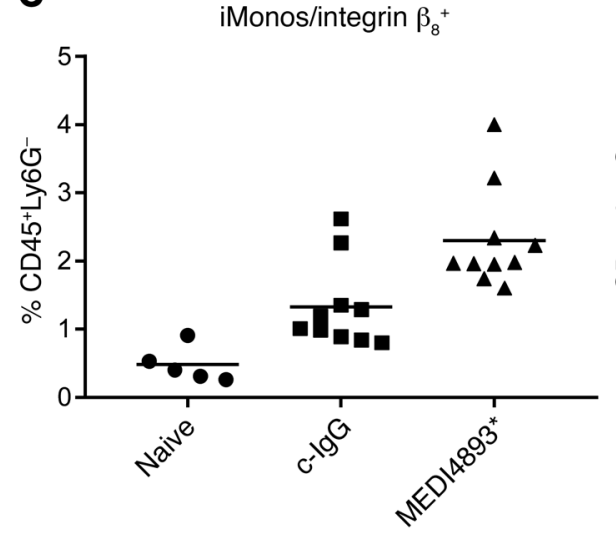

B

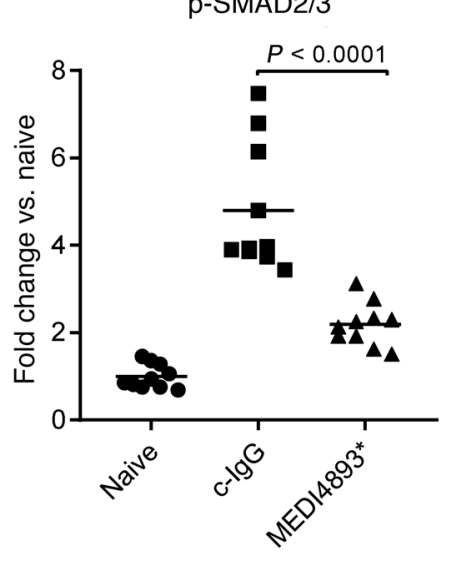

Figure 6. AT drives TGF- $\beta$ activation. (A) ELISA measuring SMAD2/3 activation in the livers of naive or 5 . aureus-infected ( $48 \mathrm{~h}$ ) C57BKS and $d b / d b$ mice. (B) ) ELISA measuring SMAD2/3 activation in the livers of naive or S. aureus-infected ( $48 \mathrm{~h}$ ) $d b / d b$ mice prophylactically treated with MEDI4893* or C-IgG. (C) FACS analysis of $\beta_{8}$ expression on inflammatory monocytes and DCs in the livers of naive or S. aureus-infected ( $48 \mathrm{~h}$ ) $d b / d b$ mice prophylactically treated with MEDI4893* or c-lgG. All data are representative of at least 3 independent experiments. $n \geq 10$ per group. Statistical analysis was determined by Kruskal-Wallis followed by Dunn's test (A-C). Bars represent the median.

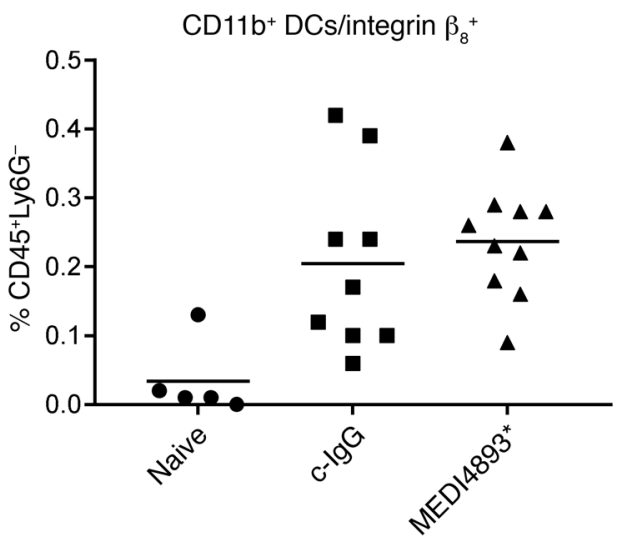

increased NET release by LDNs. We purified LDNs and HDNs from infected mice and ran gene expression as well as mass spectrometric (MS) analyses on isolated cell membranes to identify surface-expressed proteins. We found a large number of both genes and proteins that were significantly up- or downregulated in LDNs compared with expression levels in HDNs (Figure 7, A and B). Consistent with the role that TGF- $\beta$ plays in the development of LDNs, we observed increases in both gene and protein levels of TGF- $\beta$-induced protein in LDNs (data are available in the repositories Gene Expression Omnibus [GEO], GSE122195, and PRoteomics IDEntifications [PRIDE], PXD012176). Pathway analysis revealed significant upregulation in pathways such as those for platelet activation and GPCR signaling (Figure 7C). Platelet activation in response to AT has previously been shown to induce NET release by neutrophils, therefore, we focused on developing a deeper understanding of how GPCR activation could result in enhanced NET release by LDNs (17).

Ligation of $\mathrm{G}_{\mathrm{q}}$-linked GPCRs activates PLC, which leads to the degradation of $\mathrm{PIP}_{2}$ (phosphatidylinositol 4,5-biphosphate) into DAG (diacylglycerol) and $\mathrm{IP}_{3}$ (inositol trisphosphate). IP 3 induces calcium release from the ER following binding to its receptor, $\mathrm{IP}_{3} \mathrm{R}$ (Figure $7 \mathrm{E}$ ). Calcium release is required for the induction of NET release (24-26), therefore, activation of this pathway in LDNs could explain why they are prone to NET secretion. Three GPCRs were found to be upregulated in the LDNs as compared with HDNs by both MS and RNA analyses (Figure 7D, Supple- mental Figure 5, and Supplemental Table 1). Of the 3 GPCRs, only MRGPRA2a is a $\mathrm{G}_{\mathrm{q}}$-linked GPCR and capable of driving PIP deg- $_{2}$ radation. $\mathrm{PIP}_{2}$ is converted from $\mathrm{PIP}_{3}$ by the phosphatase PTEN, and loss of $\mathrm{PIP}_{3}$ can be monitored by a drop in AKT phosphorylation $(27,28)$. Analysis of membrane-associated AKT and p-AKT in LDNs as compared with HDNs revealed a significant $(P<0.0001)$ reduction of AKT activation in LDNs, consistent with a loss of $\mathrm{PIP}_{3}$ (Figure 7F). AKT phosphorylates $\mathrm{IP}_{3} \mathrm{R}$ (IP3 receptor), thereby inhibiting $\mathrm{Ca}^{2+}$ flux (29). We observed reduced phosphorylation of IP3R in LDNs as compared with HDNs, consistent with reduced AKT activity (Supplemental Figure 6). To determine whether reduced levels of p-AKT were simply due to LDNs not being activated in vivo, we exposed purified HDNs and LDNs to $N$-formylmethionyl-leucyl-phenylalanine (fMLP) ex vivo. Minimal additional activation of AKT was observed in either HDNs or LDNs, suggesting that both were maximally activated in vivo (Supplemental Figure 7). PTEN expression (both RNA and protein) was significantly $(P<0.05)$ increased in LDNs, correlating with the loss of AKT activation (Figure 7G). We decided to test whether inhibition of PTEN with the small-molecule VO-OHpic would improve the survival of diabetic mice. VO-OHpic-treated mice survived at a significantly higher rate than did control mice infected with S. aureus $(P=0.0491)$ (Figure $7 \mathrm{H})$. We conclude that increased PTEN levels in LDNs lead to an altered $\mathrm{PIP}_{2} / \mathrm{PIP}_{3}$ balance, resulting in excessive NET production, and that inhibition of PTEN can improve the survival of diabetic mice infected with $S$. aureus. 
A Surface protein LDNs vs. HDNs

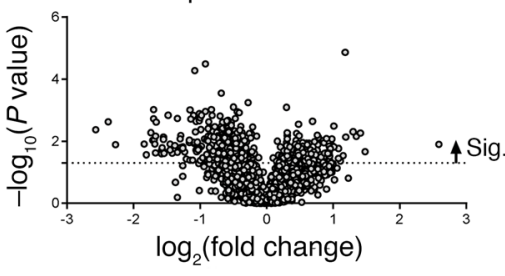

C
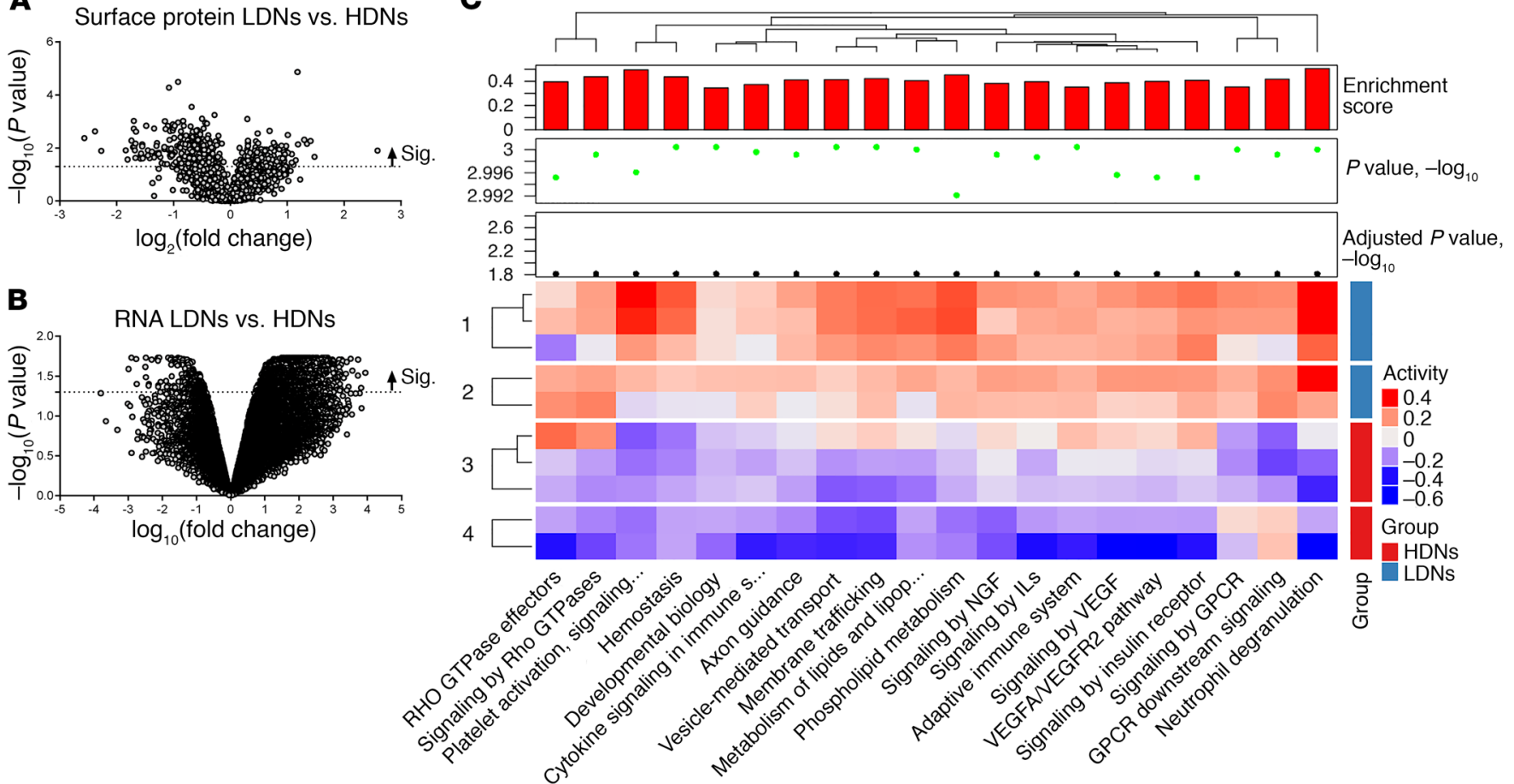

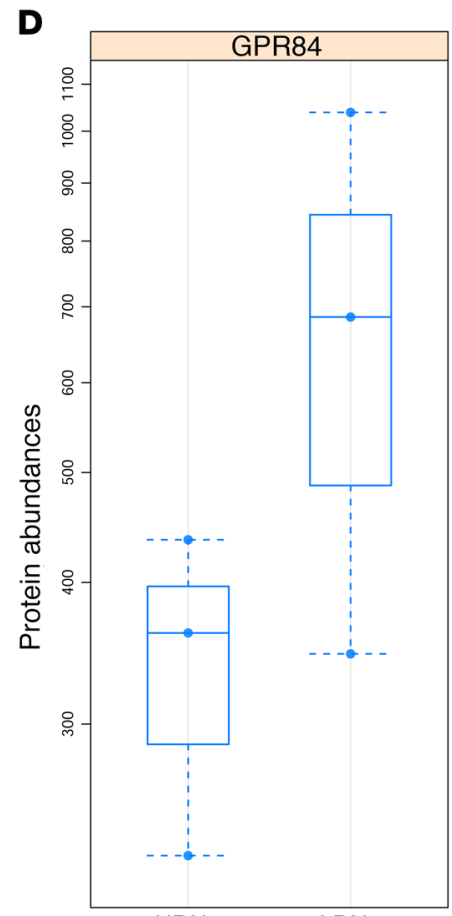

HDNs

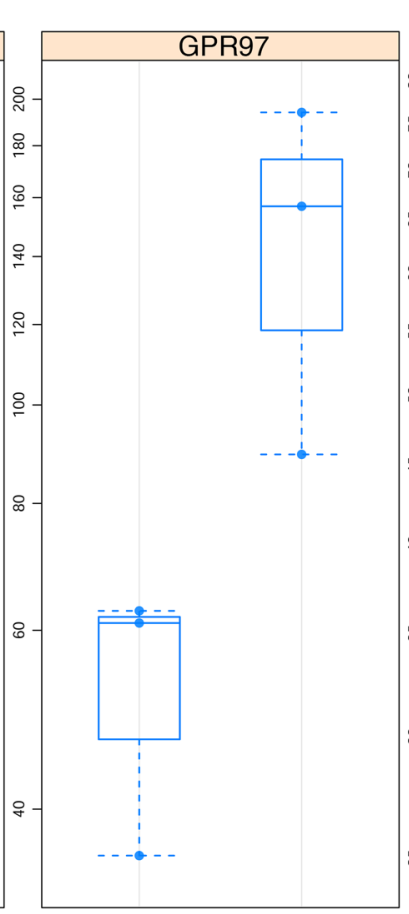

HDNs

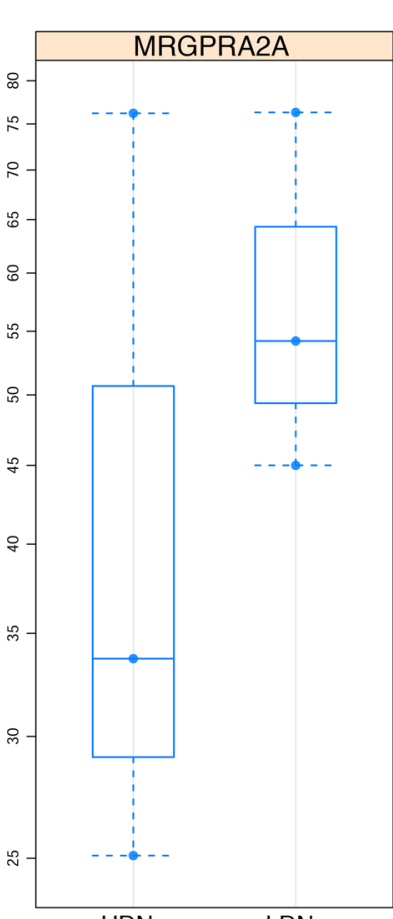

HDN
E
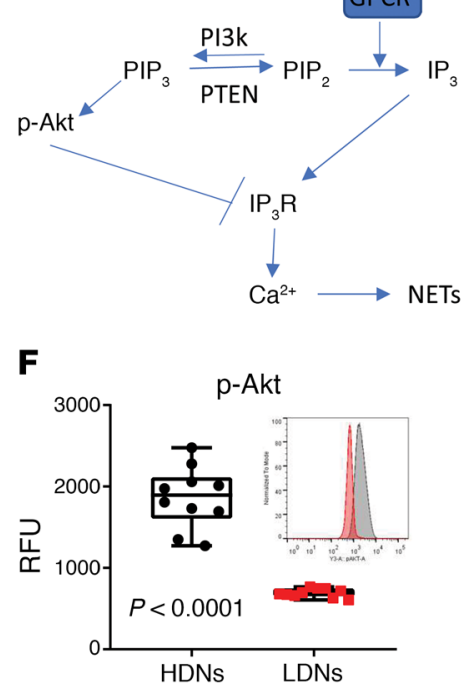

G

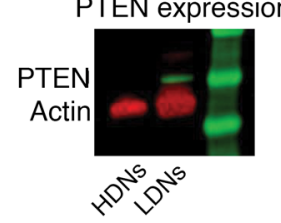

H

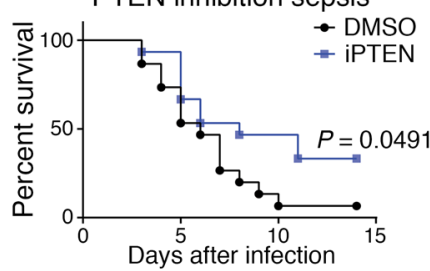


Figure 7. Altered PTEN expression in LDNs. (A) Volcano plots demonstrating significantly up- and downregulated surface proteins in LDNs as compared with HDNs. (B) Volcano plots demonstrating significantly upand downregulated RNA expression in LDNs as compared with HDNs. (C) Pathway analysis based on gene expression. (D) MS analysis of GPCRs on the surface of HDNs and LDNs. Bars show the minimum and maximum. (E) Signaling pathway altered in LDNs. (F) FACS analysis of AKT phosphorylation in HDNs and LDNs isolated from the blood of $d b / d b$ mice 48 hours after infection with S. aureus. Bars represent the median. (C) Western blot analysis of PTEN expression in HDNs and LDNs isolated from the blood of $d b / d b$ mice 48 hours after infection with S. aureus. (H) Survival of $d b / d b$ mice treated daily with the PTEN inhibitor VO-OHpic (iPTEN). All data are representative of at least 3 independent experiments. $n \geq 10$ per group. Statistical significance was determined by Mann-Whitney $U$ test (F) and log-rank test $(\mathbf{H})$.

\section{Discussion}

Diabetes is a major risk factor for severe infection with the opportunistic pathogen $S$. aureus (30). Here, we describe a unique population of neutrophils that contribute to the pathogenesis of $S$. aureus infection in diabetic mice. We demonstrate that neutralization of a key $S$. aureus virulence factor limits activation of TGF- $\beta$ signaling and subsequently reduces LDN numbers and NET release. A hallmark of these LDNs is excessive NET release, which we observed in both the liver, a key target organ of systemic infections, and the blood. PTEN regulation of the $\mathrm{PIP}_{2} / \mathrm{PIP}_{3}$ balance was shifted toward increased $\mathrm{PIP}_{2}$, which can be linked to the excessive production of NETs by LDNS (31).

The involvement of AT and NETs in the host response to S.aureushasbeendescribed previously. Neutrophilsdonotexpress appreciable levels of ADAM10, the receptor for AT, but platelets do at very high levels $(17,32)$. In response to AT, platelets aggregate and bind to neutrophils, resulting in activation of caspase-1mediated signaling and NET release $(17,33)$. Knocking out ADAM10 on platelets reduces the severity of infection in nondiabetic mice. Our data demonstrate that, in the diabetic host, AT can act through a second pathway, which alters the neutrophil phenotype and subsequentresponse to $S$.aureusinfection. In the diabetic host, AT-dependent activation of TGF- $\beta$ signaling drives expansion of LDNs. These specialized neutrophils have been shown to spontaneously release NETs in other disease contexts, and we now show that their presence correlates with NET release in a diabetic infection model (21). Comparison of LDNs with HDNs revealed an upregulation of platelet-binding proteins in the LDN population. We identified alterations in GPCR and PI3k/ PTEN signaling in LDNs, which can potentiate NET production. Therefore, AT promotes both expansion of LDNs, which spontaneously release NETs, and platelet activation, which further activates neutrophils.

Activation of TGF- $\beta$ signaling is dependent on the interaction of pro-TGF- $\beta$ and integrins, which facilitates activation of the latent complex (34). Following its activation, TGF- $\beta$ binds to its receptor complex, activating SMAD transcription factors and driving the expression of downstream genes. In this model of diabetic infection, $\alpha_{v} \beta_{6 / 8}$ integrin was primarily responsible for TGF- $\beta$-dependent LDN increases. Neutralization of either the integrin or TGF- $\beta$ prevented LDN increases and reduced animal mortality. We identified 2 populations of cells, an inflammatory monocyte population and a DC population, that expressed the $\beta_{8}$ component and were markedly increased in the infected diabetic mice. DCs were previously implicated in the activation of TGF- $\beta$ in the context of autoimmunity and parasitic infection $(22,35,36)$. Our data suggest that these cells play a central role in the pathogenesis of diabetic infection, given their ability to activate TGF- $\beta$ and promote expansion of LDNs.

Transcriptional analysis of LDNs also reflected their increased activation state as compared with HDNs. Pathway analysis demonstrated enrichment in innate immune pathways including those for phagocytic, Toll-like signaling, transendothelial migration, and inositol phosphate metabolism. PTEN plays a central role in regulating much of this signaling. Upregulated in LDNs, PTEN shunts signaling away from AKT, which is involved in chemotaxis and sensing of bacteria via TLRs (37-39). By degrading $\mathrm{PIP}_{3}$ into $\mathrm{PIP}_{2}$, PTEN decreases AKT recruitment to the cell membrane, preventing its phosphorylation, as well as enabling GPCR degradation of PIP into $\mathrm{IP}_{3}$ and activating $\mathrm{Ca}^{2+}$ signaling from the ER. When active, AKT blocks $\mathrm{IP}_{3}$ signaling through its interaction with the $\mathrm{IP}_{3} \mathrm{R}$ on the ER membrane. PTEN therefore not only increases the substrate for $\mathrm{IP}_{3} \mathrm{R}$ signaling but also limits the ability of the cell to block signaling through this receptor. Uninhibited $\mathrm{IP}_{3}$ signaling could potentially override other inhibitory pathways, such as the ability of the serum to prevent NET formation (40). PTEN has been implicated previously in NET formation, and we now link its activation to altered neutrophil function in a model of diabetic infection (31)

It is notable that we did not observe a bacterial clearance defect early in the course of infection in the diabetic mice as compared with nondiabetic control mice. The similar bacterial numbers we observed 48 hours after infection, despite significant differences in survival, highlight the contribution of excessive inflammation or an exaggerated host response to the increase in mortality. Infection in diabetic mice results in an increased and prolonged inflammatory response (41). NETs can contribute to the proinflammatory environment in a variety of diseases, including in inflammatory bowel disease, lupus, cancer, and infection (17, 42-46). The DNA backbone of the NETs activates TLR signaling, drives barrier disruption by altering endothelial and epithelial cell junctions, and prevents efferocytosis of neutrophils by other immune cells such as macrophages (47-49). NETs can also inhibit complement activation, a key component of the innate immune defense against bacterial pathogens (50). In the context of a diabetic wound, NETs contribute to delayed healing, perhaps enabling additional opportunities for infection (16). We now show that NETs correlate with increased mortality in a model of systemic infection in a diabetic host.

Diabetes is associated with an increased incidence of severe bacterial infections. We identified a neutrophil subset, previously linked to noninfectious diseases such as lupus, that is elevated during $S$. aureus infection in the diabetic host. Release of proinflammatory NETs by LDNs correlated with increased mortality. We also identify 3 potential therapeutic targets for the prevention of LDN development. Targeting a key toxin released by $S$. aureus or blocking steps in the LDN 
maturation pathway prevented LDN increases and subsequent NET induction and increased survival following infection in the diabetic host.

\section{Methods}

In vivo model of systemic infection. Frozen stock cultures of $S$. aureus USA300 strain SF8300 were thawed and diluted to the appropriate inoculum in sterile PBS, pH 7.2 (Invitrogen, Thermo Fisher Scientific) (32). Specific pathogen-free 7- to 8-week-old female BKS. Cg-Dok7 $<\mathrm{m}>+/+$ Lepr $<\mathrm{db}>/ \mathrm{J}(d b / d b)$, C57BKS, C57BL/6J - STZ, and C57BL/6J mice (The Jackson Laboratory) were briefly anesthetized and maintained in 3\% isoflurane (Butler Schein Animal Health) with oxygen at $31 / \mathrm{min}$ and infected i.v. All bacterial suspensions were administered in $100 \mu \mathrm{l}$ PBS. In select experiments, the neutralizing antibodies MEDI4893*, anti- $\alpha_{\mathrm{v}} \beta_{6 / 8}$ (clone 264RAD) (51), anti- $\alpha_{\mathrm{v}} \beta_{6}$ (clone 3G9), c-IgG (AstraZeneca), anti-TGF- $\beta$ (clone 1D11.16.8, Bio X CELL), or control mouse IgG1 were administered i.p. $(15 \mathrm{mg} / \mathrm{kg}$ ) in $0.5 \mathrm{ml} 24$ hours prior to infection. MEDI4893* and c-IgG have been previously described (32). Rosiglitazone (SigmaAldrich) was administered (10 mg/kg) orally for 7 days. Mice were infected 24 hours after the final dose of rosiglitazone. Animals were euthanized with $\mathrm{CO}_{2}$ at the indicated time points and blood, liver, or kidneys were collected for analysis. The bacterial load in the kidneys was determined by plating serial dilutions on trypticase soy agar (TSA).

ELISA. To measure NETs, a hybrid of 2 different ELISA kits was used. Plates were initially coated with anti-elastase capture antibody (R\&D Systems). Fresh serum samples were added to the coated wells and then incubated and washed. Next, anti-DNA-POD antibody (Roche) was used to detect DNA in the captured proteins in the wells. SMAD2/3 activation was measured by ELISAs for total SMAD2/3 (Cell Signaling Technology, catalog 12000C) and p-SMAD2/3 (Cell Signaling Technology, catalog 12001). Plates were developed with ABTS solution and ABTS stop solution. Absorbances were measured at $405 \mathrm{~nm}$ on a plate reader using SoftMax Pro software. SMAD2/3 activation is reported as the ratio of p-SMAD2/3 to total SMAD2/3.

$H D N$ and LDN purification. HDNs and LDNs were isolated from whole blood. Following sacrifice, blood was collected and layered over with Histopaque 1077 (Sigma-Aldrich). Cells were separated by centrifugation (500 $\mathrm{g}, 30$ minutes). The lower fraction was treated with ACK lysis buffer (Thermo Fisher Scientific) to remove red blood cells from the HDNs. The upper (PBMC) fraction was washed twice with PBS, and LDNs were isolated with the EasySep Mouse Neutrophil Enrichment Kit (STEMCELL Technologies). Purified cell populations were lysed for protein or RNA analysis as described in the Supplemental Methods. In select experiments, whole blood was stimulated with TGF- $\beta$ (10 ng/ml for 3 hours, R\&D Systems) or albumin prior to density separation. Gene array data are available in the NCBI's GEO database (GEO GSE12219). Proteomics data are available in the ProteomeXchange Consortium (PRIDE accession no. PXD012176).

Flow cytometry. The liver was minced and digested $(5 \mathrm{mg} / \mathrm{ml}$ collagenase D [Roche], $5 \mathrm{mg} / \mathrm{ml}$ DNase I [Sigma-Aldrich], $30 \mathrm{mg} / \mathrm{ml}$ dispase II [Roche]) for 45 minutes. Following digestion, the tissue was dissociated using a GentleMACS tissue dissociator (Miltenyi Biotec). Following dissociation, cells were purified by Percoll gradient. Cells were washed twice in ice-cold FACS buffer (PBS with 5\% FBS and 0.1\% sodium azide). Fc receptors were blocked with anti-mouse CD16/ CD32 (eBioscience), and cells were stained with antibodies against mouse CD45 (BV605 or BV711 conjugated, clone 30-F11; Biolegend, I-A/I-E (BV650 conjugated, clone M5/114; Biolegend), CD24 (BV711 conjugated, clone M1/69; Biolegend), CD103 (PE/Dazzle conjugated, clone 2E7; Biolegend), CD11c (APC-Cy5.5 or BV785 conjugated, clone N418; Biolegend or eBioscience), CD11b (BV605 or APC-Cy7 conjugated, clone M1/70; Biolegend or eBioscience), Ly6-G (APC or PE-Cy7 conjugated, clone 1A8, Biolegend or eBioscience), and Ly6-C (BV421 or AF488 conjugated, clone HK1.4; Biolegend). The polyclonal antibody against integrin $\beta_{8}$ was FITC conjugated (VWR). Dead cells were excluded with Live/Dead Aqua (BioLegend). Cells were imaged using the LSR II Flow Cytometer (BD Biosciences) and analyzed with FlowJo software.

Western blot analysis. Cells were lysed with RIPA buffer (Thermo Fisher Scientific) containing cOmplete protease inhibitor (SigmaAldrich) and then frozen. In select experiments, $\mathrm{IP}_{3} \mathrm{R}$ was immunoprecipitated using anti-IP $\mathrm{R}_{3}$ (Abcam, catalog ab5804) and the Dynabeads Protein G Immunoprecipitation Kit (Thermo Fisher Scientific). Equal amounts of protein were separated on $4 \%$ to $12 \%$ BisTris NuPAGE gels and transferred onto PVDF membranes (Thermo Fisher Scientific). Immunodetection was performed using anti- $\mathrm{H}_{3} \mathrm{Cit}$ (Abcam, catalog ab5103), anti-lactoferrin (Abcam, catalog ab77705), anti-MMP9 (Abcam, catalog ab38898), anti-IP ${ }_{3}$ (Abcam, catalog ab5804), anti-p-Ser/Thr (Abcam, catalog ab17464), anti-p-AKT (Cell Signaling Technology, catalog 4060), and anti-actin (SigmaAldrich, catalog A3854). Proteins were visualized with the Odyssey imaging system (Li-COR).

Confocal imaging. Livers were fixed overnight in 10\% formalin, paraffin mounted, and sectioned. Slides were washed in xylene (20 minutes) and rehydrated in sequential ethanol baths (5 minutes each in $100 \%, 95 \%, 70 \%$, and $50 \% \mathrm{EtOH}$ in $\mathrm{H}_{2} \mathrm{O}$ ). The tissue was rinsed in PBS prior to antigen retrieval $\left(0.1 \mathrm{M}\right.$ citrate buffer, $95^{\circ} \mathrm{C}, 20 \mathrm{~min}$ utes). Slides were blocked for 1 hour with BlockAid (Thermo Fisher Scientific) and stained overnight with anti-Ly6-G (BD Pharmingen, clone $1 \mathrm{~A} 8$ ) and anti- $\mathrm{H}_{3} \mathrm{Cit}$ (Abcam). Neutrophils and NETs were visualized with AF488 anti-rabbit and AF647-conjugated anti-rat IgG secondary antibodies (Molecular Probes). The slides were imaged using a Zeiss LSM 880 with an Airyscan confocal microscope (Zeiss; $\times 40$ magnification).

Neutrophil restimulation. HDNs and LDNs were purified from the blood of infected mice 48 hours after infection and incubated with $100 \mathrm{nM}$ fMLP (Sigma-Aldrich). At select time points, the cells were lysed and processed for analysis of AKT activation by Western blotting as described above.

Statistics. Data were analyzed using 2-tailed $t$ tests, MannWhitney $U$ tests, Kruskal-Wallis followed by Dunn's tests, or log-rank tests where appropriate. All statistical analyses were performed using GraphPad Prism, version 6.0. Sample sizes for all animal studies were estimated using a log-rank test with a $5 \%$ type I error rate and $80 \%$ power. The hypothesized effect size for each comparison was derived from historical data or pilot study data. Sample sizes were calculated using nQuery Advisor 7.0 software. All animals were randomly assigned to treatment groups using an online randomization tool implemented in Microsoft Excel. A P value of 0.05 or less was considered statistically significant. 
Study approval. All animal studies were approved by the MedImmune Institutional Animal Care and Use Committee and were conducted in an Association for Accreditation and Assessment Laboratory Animal Care-accredited (AAALAC-accredited) facility in compliance with US regulations governing the housing and use of animals.

\section{Author contributions}

TSC designed and performed the research, analyzed the data, and wrote the manuscript. VT, JB, OJN, and JJW designed and per- formed the research. AT, RC, WY, and YL analyzed the data. RR, $\mathrm{SH}, \mathrm{CKS}, \mathrm{MAT}$, and BRS wrote the manuscript.

\section{Acknowledgments}

All funding for this study was provided by AstraZeneca.

Address correspondence to: Taylor S. Cohen, One Medimmune Way, Gaithersburg, Maryland 20878, USA. Phone: 301.398.2405; Email:cohent@medimmune.com.
1. Joshi N, Caputo GM, Weitekamp MR, Karchmer AW. Infections in patients with diabetes mellitus. N Engl J Med.1999;341(25):1906-1912.

2. Smit J, Søgaard M, Schønheyder HC, Nielsen H, Frøslev T, Thomsen RW. Diabetes and risk of community-acquired Staphylococcus aureus bacteremia: a population-based case-control study. Eur JEndocrinol. 2016;174(5):631-639.

3. Tuchscherr L, et al. Staphylococcus aureus requires less virulence to establish an infection in diabetic hosts. Int J Med Microbiol. 2018;308(7):761-769.

4. Rich J, Lee JC. The pathogenesis of Staphylococcus aureus infection in the diabetic NOD mouse. Diabetes. 2005;54(10):2904-2910.

5. Scully IL, et al. Neutrophil killing of Staphylococcus aureus in diabetes, obesity and metabolic syndrome: a prospective cellular surveillance study. Diabetol Metab Syndr. 2017;9:76.

6. Marhoffer W, Stein M, Schleinkofer L, Federlin K. Evidence of ex vivo and in vitro impaired neutrophil oxidative burst and phagocytic capacity in type 1 diabetes mellitus. Diabetes Res Clin Pract. 1993;19(3):183-188.

7. Hanses F, Park S, Rich J, Lee JC. Reduced neutrophil apoptosis in diabetic mice during staphylococcal infection leads to prolonged $\operatorname{Tnf} \alpha$ production and reduced neutrophil clearance. PLOS ONE. 2011;6(8):e23633.

8. Sørensen OE, Borregaard N. Neutrophil extracellular traps - the dark side of neutrophils. JClin Invest. 2016;126(5):1612-1620.

9. Brinkmann V, Zychlinsky A. Beneficial suicide: why neutrophils die to make NETs. Nat Rev Microbiol. 2007;5(8):577-582.

10. Malachowa N, Kobayashi SD, Quinn MT, DeLeo FR. NET Confusion. Front Immunol. 2016;7:259.

11. Martinod K, et al. PAD4-deficiency does not affect bacteremia in polymicrobial sepsis and ameliorates endotoxemic shock. Blood. 2015;125(12):1948-1956.

12. Menegazzi R, Decleva E, Dri P. Killing by neutrophil extracellular traps: fact or folklore? Blood. 2012;119(5):1214-1216

13. Ortines RV, et al. Neutralizing Alpha-Toxin Accelerates Healing of Staphylococcus aureus-Infected Wounds in Nondiabetic and Diabetic Mice. Antimicrob Agents Chemother. 2018;62(3):e02288-17.

14. Carestia A, et al. NETosis before and after Hyperglycemic Control in Type 2 Diabetes Mellitus Patients. PLoS ONE. 2016;11(12):e0168647.

15. Fadini GP, et al. NETosis Delays Diabetic Wound Healing in Mice and Humans. Diabetes. 2016;65(4):1061-1071.

16. Wong SL, et al. Diabetes primes neutrophils to undergo NETosis, which impairs wound healing. Nat Med. 2015;21(7):815-819.

17. Powers ME, Becker RE, Sailer A, Turner JR, Bubeck Wardenburg J. Synergistic action of Staphylococcus aureus $\alpha$-toxin on platelets and myeloid lineage cells contributes to Lethal Sepsis. Cell Host Microbe. 2015;17(6):775-787.

18. Tsuda Y, Takahashi H, Kobayashi M, Hanafusa T, Herndon DN, Suzuki F. Three different neutrophil subsets exhibited in mice with different susceptibilities to infection by methicillinresistant Staphylococcus aureus. Immunity. 2004;21(2):215-226.

19. Sagiv JY, et al. Phenotypic diversity and plasticity in circulating neutrophil subpopulations in cancer. Cell Rep. 2015;10(4):562-573.

20. Fridlender ZG, et al. Polarization of tumorassociated neutrophil phenotype by TGF-beta: "N1" versus "N2" TAN. Cancer Cell. 2009;16(3):183-194.

21. Villanueva $E$, et al. Netting neutrophils induce endothelial damage, infiltrate tissues, and expose immunostimulatory molecules in systemic lupus erythematosus. J Immunol. 2011;187(1):538-552.

22. Travis MA, et al. Loss of integrin alpha(v)beta8 on dendritic cells causes autoimmunity and colitis in mice. Nature. 2007;449(7160):361-365.

23. Heldin CH, Miyazono K, ten Dijke P. TGF-beta signalling from cell membrane to nucleus through SMAD proteins. Nature. 1997;390(6659):465-471.

24. Douda DN, Khan MA, Grasemann H, Palaniyar N. SK3 channel and mitochondrial ROS mediate NADPH oxidase-independent NETosis induced by calcium influx. Proc Natl Acad Sci U S A. 2015;112(9):2817-2822.

25. Zhou Y, et al. Evidence for a direct link between PAD4-mediated citrullination and the oxidative burst in human neutrophils. Sci Rep. 2018;8(1):15228.

26. Gupta AK, Giaglis S, Hasler P, Hahn S. Efficient neutrophil extracellular trap induction requires mobilization of both intracellular and extracellular calcium pools and is modulated by cyclosporine $\mathrm{A}$. PLOS ONE. 2014;9(5):e97088.

27. Marte BM, Downward J. PKB/Akt: connecting phosphoinositide 3-kinase to cell survival and beyond. Trends Biochem Sci. 1997;22(9):355-358.

28. Stephens $L$, et al. Protein kinase B kinases that mediate phosphatidylinositol 3,4,5-trisphosphatedependent activation of protein kinase B. Science. 1998;279(5351):710-714

29. Szado T, et al. Phosphorylation of inositol 1,4,5-trisphosphate receptors by protein kinase
B/Akt inhibits Ca2+ release and apoptosis. Proc Natl Acad Sci U S A. 2008;105(7):2427-2432.

30. Hansen MU, et al. Diabetes increases the risk of disease and death due to Staphylococcus aureus bacteremia. A matched case-control and cohort study. Infect Dis (Lond). 2017;49(9):689-697.

31. Teimourian S, Moghanloo E. Role of PTEN in neutrophil extracellular trap formation. Mol Immunol. 2015;66(2):319-324.

32. Cohen TS, et al. Staphylococcus aureus $\alpha$ toxin potentiates opportunistic bacterial lung infections. Sci Transl Med. 2016;8(329):329ra31.

33. Surewaard BGJ, et al. $\alpha$-Toxin induces platelet aggregation and liver injury during Staphylococcus aureus sepsis. Cell Host Microbe. 2018;24(2):271-284.e3.

34. Worthington JJ, Klementowicz JE, Travis MA. TGF : a sleeping giant awoken by integrins. Trends Biochem Sci. 2011;36(1):47-54.

35. Worthington JJ, et al. Loss of the TGF $\beta$ activating integrin $\alpha v \beta 8$ on dendritic cells protects mice from chronic intestinal parasitic infection via control of type 2 immunity. PLoS Pathog. 2013;9(10):e1003675.

36. Fenton TM, et al. Inflammatory cues enhance TGF $\beta$ activation by distinct subsets of human intestinal dendritic cells via integrin $\alpha v \beta 8$. Mucosal Immunol. 2017;10(3):624-634.

37. Barati MT, Scherzer J, Wu R, Rane MJ, Klein JB. Cytoskeletal rearrangement and Src and PI-3K-dependent Akt activation control GABA(B)R-mediated chemotaxis. Cell Signal. 2015;27(6):1178-1185

38. Kumar S, et al. The small GTPase Rap1b negatively regulates neutrophil chemotaxis and transcellular diapedesis by inhibiting Akt activation. JExp Med. 2014;211(9):1741-1758.

39. Strassheim D, et al. Phosphoinositide 3-kinase and Akt occupy central roles in inflammatory responses of Toll-like receptor 2-stimulated neutrophils. JImmunol. 2004;172(9):5727-5733.

40. Fuchs TA, et al. Novel cell death program leads to neutrophil extracellular traps. JCell Biol. 2007;176(2):231-241

41. Nielsen TB, et al. Diabetes exacerbates infection via hyperinflammation by signaling through TLR4 and RAGE. MBio. 2017;8(4):e00818-17.

42. Yazdani HO, et al. IL-33 exacerbates liver sterile inflammation by amplifying neutrophil extracellular trap formation. J Hepatol. 2018;68(1):130-139.

43. He Z, et al. Phosphotidylserine exposure and neutrophil extracellular traps enhance procoagulant activity in patients with inflammatory bowel disease. Thromb Haemost. 2016;115(4):738-751. 
44. Lood C, et al. Neutrophil extracellular traps enriched in oxidized mitochondrial DNA are interferogenic and contribute to lupus-like disease. Nat Med. 2016;22(2):146-153.

45. Albrengues J, et al. Neutrophil extracellular traps produced during inflammation awaken dormant cancer cells in mice. Science. 2018;361(6409):eaao4227.

46. Jorch SK, Kubes P. An emerging role for neutrophil extracellular traps in noninfectious disease.
Nat Med. 2017;23(3):279-287.

47. Huang $\mathrm{H}$, et al. Damage-associated molecular pattern-activated neutrophil extracellular trap exacerbates sterile inflammatory liver injury. Hepatology. 2015;62(2):600-614.

48. Grégoire M, et al. Impaired efferocytosis and neutrophil extracellular trap clearance by macrophages in ARDS. Eur Respir J. 2018;52(2):1702590.

49. Pieterse E, et al. Neutrophil extracellular traps drive endothelial-to-mesenchymal transition. Arterioscler Thromb Vasc Biol. 2017;37(7):1371-1379.

50. Qaddoori Y, et al. Extracellular Histones Inhibit Complement Activation through Interacting with Complement Component 4. JImmunol. 2018;200(12):4125-4133.

51. Eberlein C, et al. A human monoclonal antibody 264RAD targeting av $\beta 6$ integrin reduces tumour growth and metastasis, and modulates key biomarkers in vivo. Oncogene. 2013;32(37):4406-4416. 